\title{
Economic Analysis of Lagrangian and Genetic Algorithm for the Optimal Capacity Planning of Photovoltaic Generation
}

\author{
Jeeng-Min Ling and Ping-Hsun Liu \\ Department of Electrical Engineering, Southern Taiwan University of Science and Technology, Tainan 710, Taiwan \\ Correspondence should be addressed to Jeeng-Min Ling; jmling@mail.stust.edu.tw
}

Received 25 August 2014; Accepted 28 September 2014

Academic Editor: Teen-Hang Meen

Copyright ( 2015 J.-M. Ling and P.-H. Liu. This is an open access article distributed under the Creative Commons Attribution License, which permits unrestricted use, distribution, and reproduction in any medium, provided the original work is properly cited.

\begin{abstract}
The optimal allocation problem for a stand-alone photovoltaic (SPV) generation can be achieved by good compromise between system objective and constraint requirements. The Lagrange technique (LGT) is a traditional method to solve such constrained optimization problem. To consider the nonlinear features of reliability constraints evolving from the consideration of different scenarios, including variations of component cost, load profile and installation location, the implementation of SPV generation planning is time-consuming and conventionally implemented by a probability method. Genetic Algorithm (GA) has been successfully applied to many optimization problems. For the optimal allocation of photovoltaic and battery devices, the cost function minimization is implemented by GA to attain global optimum with relative computation simplicity. Analytical comparisons between the results from LGT and GA were investigated and the performance of simulation was discussed. Different planning scenarios show that GA performs better than the Lagrange optimization technique.
\end{abstract}

\section{Introduction}

Recently, a vast number of population based search and optimization algorithms have been applied successfully to many power and energy applications $[1,2]$. One of the popular and excellent population based algorithms is the GA; it has been developed to imitate the evolutionary principle of natural genetics [3]. GA technique conceived by Holland in the 1975 was based on a Darwinian survival-of-the-fittest strategy with sexual reproduction. Stronger individuals in the population have a higher chance of creating a better offspring. GA can be implemented as a computerized optimization searching among possible solutions as strings of ones and zeros; each iteration of simulation begins with a randomly selected population of chromosomes represented by strings.

The probabilistic process of crossover and mutation ensures that GA can explore new features that may not be in the present population. It searches the entire reachable space despite the finite population size. GA is advantageous over other search algorithms since it is less likely to be trapped by local minimum and provides a better global optimal solution $[3,4]$.
This paper studies the optimal capacity planning for a SPV generation system. Solving this nonlinear constrained optimization problem requires computing effort which increases with the size of the problem. A traditional optimization technique demonstrates a number of difficulties when faced with complex engineering problems. The major issue arises when one popular algorithm is applied to solve a number of different problems [2]. Each classical optimization algorithm should be well designed to solve only particular problems efficiently. For a system's designer and planner, the methods have the feasibility to solve different types of problems with a global perspective, do not often get converged to a locally optimal solution, and are welcome.

Many bio-inspired optimization techniques have been employed to find an adequate solution to the complicated engineering problems [1]. The aim of these techniques is to find sufficient "good" solution efficiently with the characteristic of the problem, instead of the global optimum solution, and thus it also provides attractive alternative for the large scale applications. Among these methods, GA achieves both local exploration and global exploitation to provide a robust and efficient methodology in searching a near-optimal 
solution; it has been applied to a variety of optimization applications. The potential of the GA technique applied to design a renewable energy system has been recently reported [5-9].

Several software tools are available for the design of a PV system $[5,10]$. However, most of these tools only identify and simulate a single design option; a range of possible design options are unavailable $[10,11]$. Furthermore, the impacts on the effects of nonlinearity and optimization in system model and the variations of the significant design variables are needed to investigate the effectiveness of these simulation and optimization tools applied to specific applications [12,13].

The objective of this paper is to compare the accuracy and relative performance based on GA and Lagrangian technique in the capacity planning. The best compromise between the reliability and installed cost of SPV system is investigated. Compared with the conventional Lagrangian relaxation optimization, GA can find the global optimal solutions more efficiently.

\section{The Optimal Sizing Problems}

The sizing of SPV systems is an important part of system design and planning and remains an active area for research. Sizing procedures consider the relationships between energy deliveries to load and its reliability of supply which can be tolerated by the user.

2.1. The Reliability Analysis of a SPV Generation. To access the available solar generation of a PV system in candidate region is one of the most important parameters before installation. Because of the intermittent solar radiation characteristic, power reliability analysis has been considered an important step in any power system planning and design process.

Reliability index evaluated in the simulation is the total loss of load hours (LOLH) over a specified time (usually one year). LOLH is a feasible measure of the system performance for assumed load profile. LOLH equal to 0 means that the load will always be satisfied within the specific simulation time period. Larger LOLH means that customer will suffer from a higher probability of losing power. LOLH can be defined as follows:

$$
\mathrm{LOLH}=\sum_{i=1}^{n} \sum_{j=1}^{24} f(i, j)
$$

$$
\begin{aligned}
& f(i, j) \\
& = \begin{cases}0 & \text { if }(S(i, j)-L(i, j)) \geq S_{\text {min }} \\
1-\frac{S(i, j)-S_{\text {min }}}{L(i, j)} & \text { if } S(i, j)>S_{\min }, S(i, j-1)>S_{\min } \\
1 & \text { if }(S(i, j)-L(i, j))<S_{\text {min }} \\
& \text { or } S(i, j-1)<S_{\text {min }}\end{cases}
\end{aligned}
$$

where $S(i, j)$ is the capacity state of BTY in the $i$ th day- $j$ th hour, $L(i, j)$ is the consumed load in the $i$ th day- $j$ th hour, $f(i, j)$ is system shortage in the $i$ th day- $j$ th hours, and $S_{\min }$ is minimum battery discharge capacity.

The amount of solar radiation implies the current output of a PV generation. After considering load profile, the output current of a PV generation can be conducted to evaluate the charge/discharge current of BTY $\left(I_{b}\right)$. Two main directions of $I_{b}$ lead to different operation mode of SPV: positive sign shows the mode of PV generation greater than load, while negative sign is induced by the shortage of a SPV generation. The flowchart of evaluation LOLH is shown in Figure 1.

\subsection{The Constrained Optimization Problem}

2.2.1. Objective Function. A constrained optimization problem should be considered to determine the optimal size in a SPV system. The optimal solution can make the best compromise between the two significant issues: the system power reliability and system installed cost. The objective function of the optimal capacity planning problem is to minimize the total installed cost while satisfying the reliability requirements of the SPV system. The objective function can be expressed as the installed cost of a SPV system:

$$
C=C_{\mathrm{pv}} \cdot \mathrm{PV}+C_{b} \cdot \mathrm{BTY}+C_{i}
$$

where $C$ is the total cost for installed a SPV system, $C_{i}$ is the initial cost for system installation, $\mathrm{PV} / \mathrm{BTY}$ is the capacity of solar array and battery, and $C_{\mathrm{pv}}$ and $C_{b}$ are the unit cost of $\mathrm{PV}$ $\left(\$ / \mathrm{W}_{\mathrm{p}}\right)$ and BTY $(\$ / \mathrm{Wh})$.

2.2.2. Constrained Function. After implementing the LOLH flowchart, the combinations of different PV and battery size can be conducted by a nonlinear function. Constrained function has been produced for eight different values of LOLH, 0, 10, 20, 50, 100, 150, 200, and 400 hours under different reliability requirement, in terms of different load profile. It is significant for a SPV planner to get options under different requirement of system shortages. Different constrained function with different LOLH values can be derived by a polynomial regression technique. Saber proved that if a polynomial equation is for more than six orders, the regression coefficient matrix will be in a bad condition; it means that small data variation causes relatively large parameter estimation error [14].

\section{Methodology of the Optimization Model}

3.1. Lagrangian Technique. The method of Lagrange multipliers is a popular and conventional technique for constrained optimization. The capacity combination of PV/BTY under specified system's reliability requirement can be described by the six-order polynomial constraint function $\mathbf{H}$ shown below:

$$
\begin{aligned}
\mathbf{H}= & \mathbf{f}(\mathrm{PV}, \mathrm{BTY}) \\
= & \mathrm{PV}-\left(\mathrm{p}_{1} \mathrm{BTY}+\mathrm{p}_{2} \mathrm{BTY}^{2}+\mathrm{p}_{3} \mathrm{BTY}^{3}+\mathrm{p}_{4} \mathrm{BTY}^{4}\right. \\
& \left.+\mathrm{p}_{5} \mathrm{BTY}^{5}+\mathrm{p}_{6} \mathrm{BTY}^{6}\right) .
\end{aligned}
$$




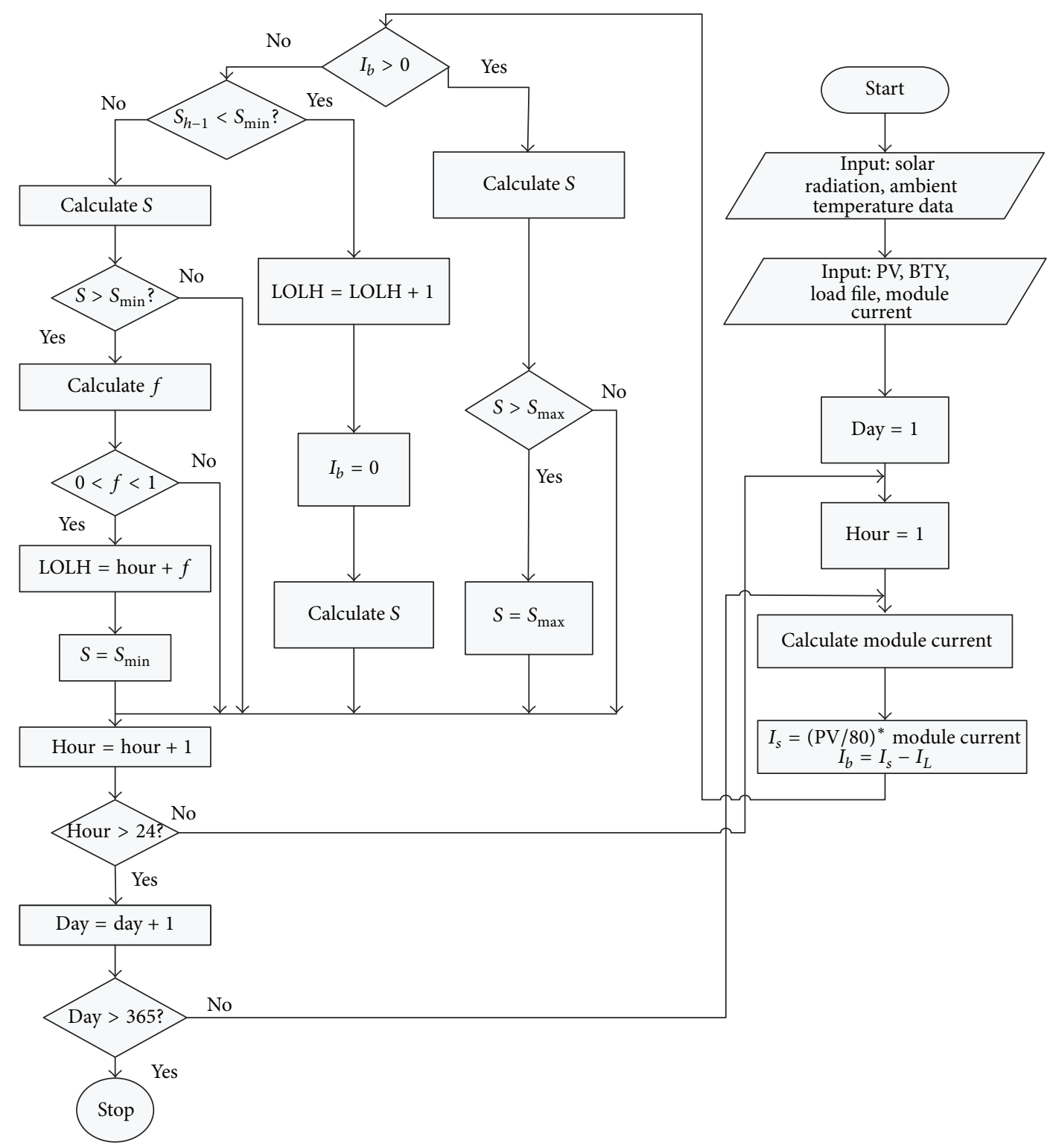

FIGURE 1: Flowchart of the reliability analysis for a SPV system.

The constrained optimization problem can be formulated from the Lagrange equation $\mathbf{L}$ after integrating the objective cost function $(\mathbf{C})$ and the constraint function $(\mathbf{H})$ in terms of an undetermined multiplier $\lambda$ :

$$
\mathbf{L}=\mathbf{C}+\lambda \mathbf{H}
$$

The optimum point occurs at the partial derivative of $\mathbf{L}$ with respect to each of the independent decision variables, that is, PV, BTY, and $\lambda$, equal to zero. Lagrange calculation uses analytical derivatives; it may not be efficient in handling discrete variables but using approximate function to get derivative. Much complicated iteration processes have implemented to trade off different Lagrange multipliers by the Lagrangian relaxation method in many engineering applications. However, the constrained optimization problem easily suffers to find critical points of the Lagrange multiplier and traps into local optimum.

3.2. GA Technique. Renewable energy planning problem includes economical objective, and it requires the assessment of long-term system performance to reach the best compromise between system reliability and cost. The minimization of the fitness or cost function is implemented by the GA method to search the optimal size of photovoltaic and battery storage devices.

Three important steps, including selection, crossover, and mutation, are to imitate nature evolution processes with GA technique. The optimal solution imposes on the selection of crossover and mutation operation; it produces the next generation when convergent criterion is satisfied. The ability of directing the random search of a GA by selecting the fittest 


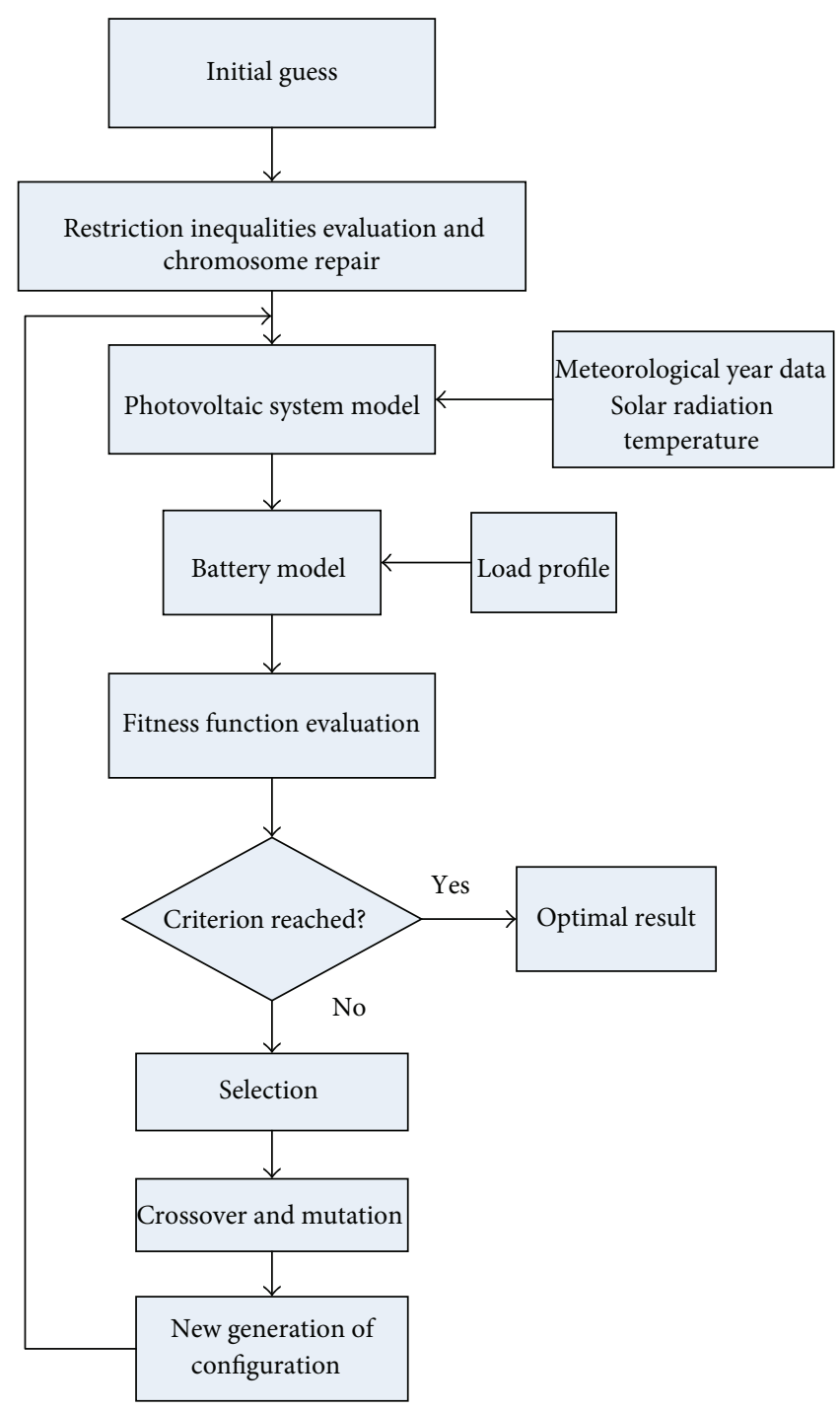

Figure 2: Flowchart of the optimal sizing simulation using GA.

chromosomes among the population is one of significant aspects during computational simulation. The optimization process of GA is iterated if any of the initial population chromosomes violates the system constraints until another new chromosome is selected.

In this study, the proposed GA technique was implemented by Matlab (trademark of the MathWorks); it employed the operators of roulette-wheel random selection, single-point crossover, and mutation, then elite replacement. Only the result of best experiment instance is presented hereafter. The GA model obtains the optimal size in terms of various degrees of reliability. Binary coded GA was introduced to solve the optimal capacity of PV and BTY. Input data includes hourly data per year, solar radiation on the horizontal surface, ambient air temperature, and load power consumption. The flowchart of the GA process applied to
SPV sizing problem is illustrated in Figure 2. The following parameters are used in the GA simulation:

(i) the population size: 100 ,

(ii) crossover rate: 0.95 ,

(iii) mutation rate: 0.05 .

\section{Analysis of Reliability and Optimal Simulation}

4.1. Reliability Simulation. The optimal sizes of a SPV system at two selected sites (Tainan and Dawu) of weather station in Taiwan were investigated and compared. Using the real meteorological data of specific year at Dawu weather station, the possible combination of PV/BTY capacity associated 


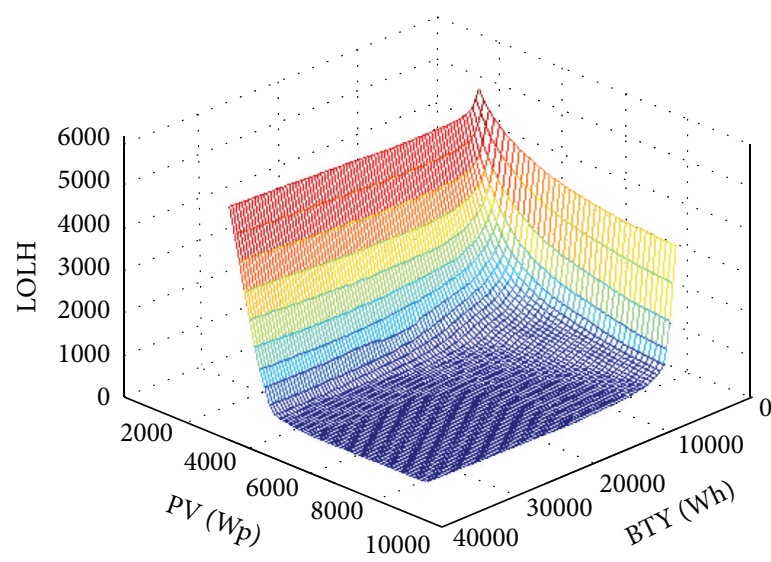

(a) Three-dimensional curves

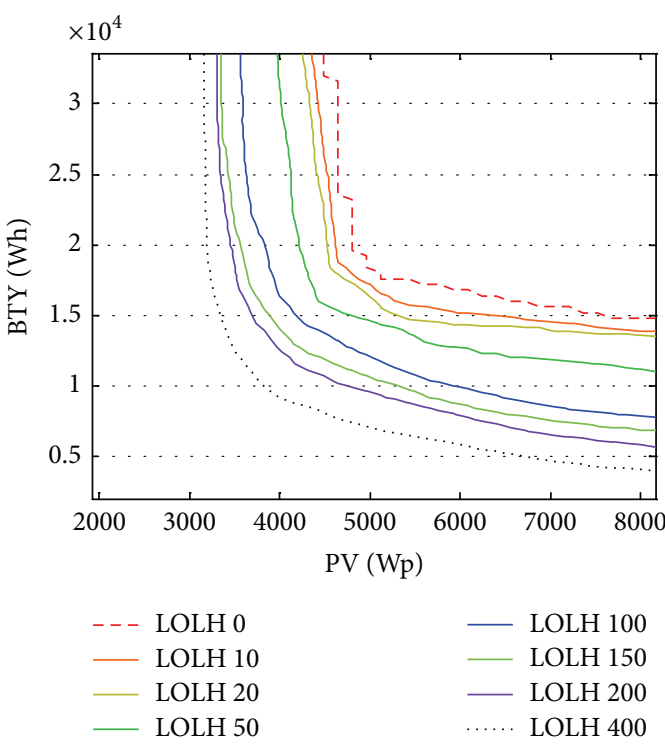

(b) Two-dimensional curves

FIGURE 3: Demonstration of PV/BTY capacity with different LOLH requirements.



(a) Chiayi

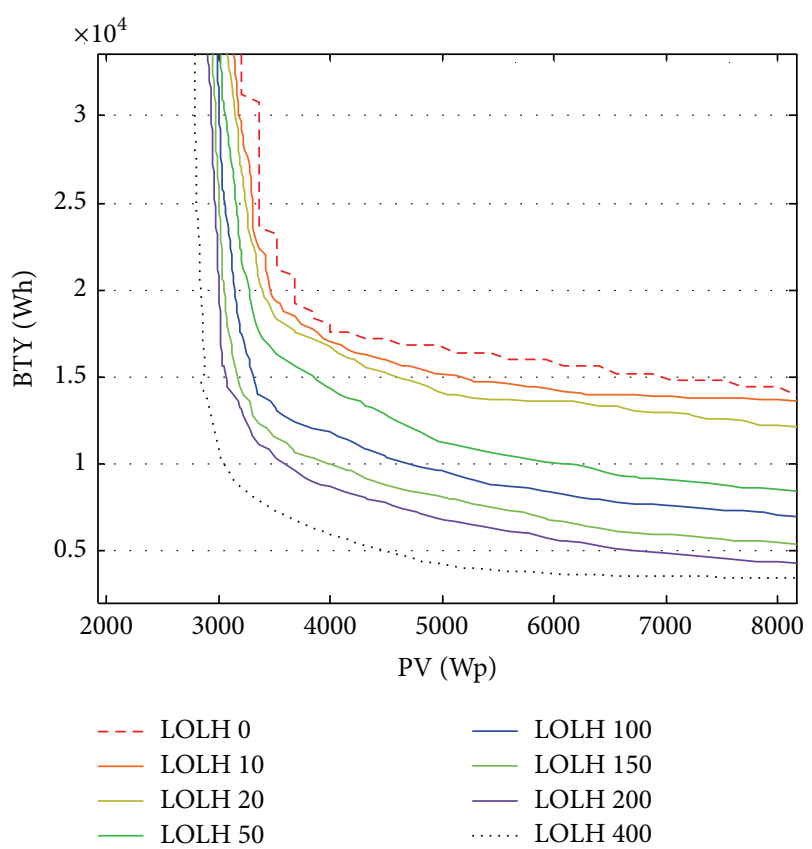

(b) Tainan

FIGURE 4: PV/BTY capacity allocations for different LOLH requirements at two locations with actual load simulation.

with different values of LOLH can be depicted by a threedimensional (3D) curve shown in the left side of Figure 3. Eight specified values of $\operatorname{LOLH}(0,10,20,50,100,150,200$, and 400 hours) are selected and depicted by two-dimensional (2D) curves with different colors in the right side of Figures 3 (b) and 4.
Each 2D curve indicates the trend of PV/BTY size changing with a constant system shortage. The different combinations of PV/BTY capacity which meet the same reliability degree of power supply can be clearly expressed by plotting the $2 \mathrm{D}$ trade-off curve. Using the eight LOLH curves shown in Figures 3(b) and 4, the influence of LOLH on the planning 
TABLE 1: Comparison between Genetic Algorithm and Lagrange technique.

(a) Tainan

\begin{tabular}{|c|c|c|c|c|c|c|c|c|}
\hline \multirow{2}{*}{ LOLH } & \multicolumn{4}{|c|}{ Genetic Algorithm } & \multicolumn{4}{|c|}{ Lagrange technique } \\
\hline & PV (Wp) & BTY (W) & Cost $(\$)$ & Time (s) & PV (Wp) & BTY (W) & Cost $(\$)$ & Time (s) \\
\hline 0 & 3487.68 & 20774.59 & 18739.35 & \multirow{8}{*}{6.63} & 3195.1 & 21702.7 & 17720.4 & \multirow{8}{*}{12.3} \\
\hline 10 & 3382.42 & 22053.13 & 18568.23 & & 3053.9 & 21063.3 & 17003.4 & \\
\hline 20 & 3252.19 & 22932.70 & 18211.16 & & 3000.6 & 20136.9 & 16590.8 & \\
\hline 50 & 3242.57 & 20085.64 & 17581.96 & & 3386.8 & 15807.1 & 17293.3 & \\
\hline 100 & 3240.58 & 16129.99 & 16754.90 & & 3201.9 & 15354.2 & 16434.3 & \\
\hline 150 & 3146.45 & 14698.85 & 16068.99 & & 3136.6 & 14913.6 & 16072.7 & \\
\hline 200 & 3120.14 & 13807.39 & 15775.51 & & 2936.5 & 18437.3 & 15973.5 & \\
\hline 400 & 3040.25 & 10242.27 & 14706.81 & & 3023.9 & 8696.6 & 14319.2 & \\
\hline
\end{tabular}

(b) Dawu

\begin{tabular}{|c|c|c|c|c|c|c|c|c|}
\hline \multirow{2}{*}{ LOLH } & \multicolumn{4}{|c|}{ Genetic Algorithm } & \multicolumn{4}{|c|}{ Lagrange technique } \\
\hline & PV (Wp) & BTY (W) & Cost $(\$)$ & Time (s) & PV (Wp) & BTY (W) & Cost $(\$)$ & Time (s) \\
\hline 0 & 4743.17 & 20581.04 & 23897.02 & \multirow{8}{*}{6.67} & 4153.9 & 21103.6 & 21565.8 & \multirow{8}{*}{12.3} \\
\hline 10 & 4692.96 & 18588.33 & 23276.66 & & 3738.6 & 20531.5 & 19728.2 & \\
\hline 20 & 4657.65 & 17350.52 & 22874.25 & & 3628.9 & 20207.4 & 19206.9 & \\
\hline 50 & 4259.91 & 18621.41 & 21490.67 & & 3830.7 & 19266.4 & 19847.3 & \\
\hline 100 & 3827.22 & 19234.76 & 19826.29 & & 3907.0 & 16408.4 & 19571.6 & \\
\hline 150 & 3643.09 & 17798.25 & 18766.64 & & 3625.3 & 17632.9 & 18658.8 & \\
\hline 200 & 3581.59 & 16314.60 & 18204.94 & & 3654.9 & 12983.0 & 17818.7 & \\
\hline 400 & 3362.52 & 14163.70 & 16852.74 & & 3172.9 & 18352.8 & 16935.2 & \\
\hline
\end{tabular}

of PV/BTY capacity of a SPV system can be identified. Considerable installed PV and BTY capacity reduces when LOLH varies from 0 to 400 hours. Different location has different solar radiation. In order to clarify the influence of locations, the meteorological data from two different weather stations in the Central Weather Bureau of Taiwan were simulated.

4.2. Optimal Sizing Simulation. The optimal solution of a SPV system planning occurs at the turning point of a LOLH curve. Analysis of the relationship of PV/BTY capacity in terms of LOLH can determine the optimal capacity allocation status. As the unit cost of a PV component is much larger than that of BTY, the total installation cost of PV significantly dominates the final optimal cost. The real solar radiation/temperature data from the central weather Bureau of Taiwan on specific year have been simulated. The actual load is the power consumption of a laboratory located at the building of the Southern Taiwan University of Science and Technology. Comparisons with optimal PV/BTY allocation corresponding to eight different LOLH at two sites are shown in Table 1.

For the LGT, the shaded cells in Table 1 indicate that the unreasonable installed cost solution is obtained. The LGT often gets worse solutions than using GA because the LGT tends to trap at a local optimal solution while GA attains a global solution. From the results of Table 1, the total installed cost of SPV system from LGT may be smaller than GA. This results from a small capacity of the testing system with an insufficient search space, while GA may perform in a poor manner [15]. For the SPV planning, larger size of system capacity and load profile entail wide searching space of possible solution. The proposed GA model can be achieved near-optimal solution under comprehensive planning requirements.

From the results shown in Figure 5, LGT may be less sensitive than GA method. Statistically, it can be proved that the performance of GA approach is slightly superior for the capacity planning of SPV system. This implied the searching technique used in the GA can be explored globally more than the LGT. According to the results, it is concluded that the local heuristic plays an important role in GA process. High quality heuristic solution can help the GA to improve its performance by reducing the likelihood of its premature convergence.

GA is achieved efficiently by a shorter computational time from the simulation result of Table 1 . It can be recognized that GA gains its advancement in the applications of complicated optimization-constrained problem, for example, photovoltaic allocation planning, where the searching of the global optimum is difficult task. Due to the probabilistic development of solutions, GA is not restricted by local optimum; it can find the global optimum system configuration with relative computational simplicity compared to conventional optimization methods such as LGT.

\section{Conclusion}

The Lagrange technique and GA applied to optimal capacity planning of SPV system are investigated. Comparison of performance was made on the minimum total installed cost of a SPV system with specified demand load and system 


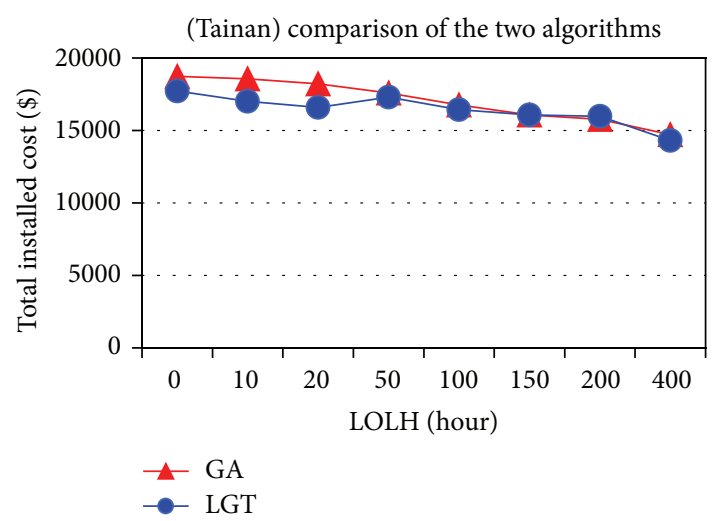

(a) Tainan

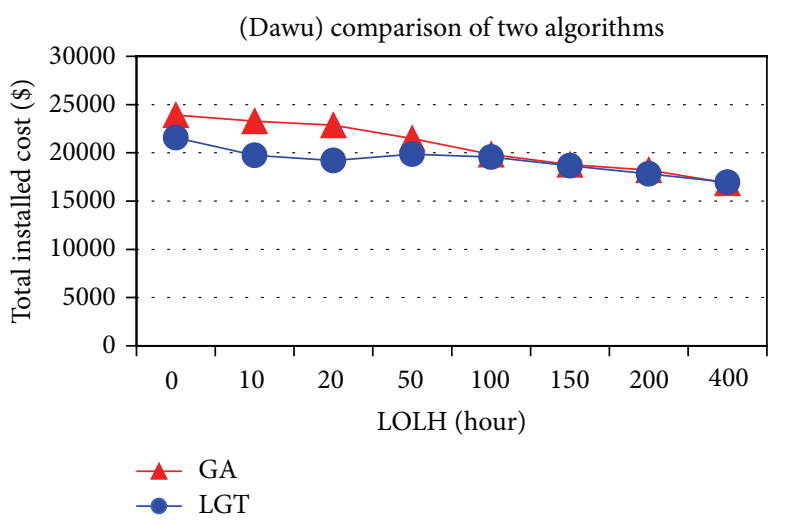

(b) Dawu

FIGURE 5: Comparisons between Genetic Algorithm and Lagrange algorithm.

reliability requirements. The simulation results show that GA is slightly superior for finding the optimal capacity planning of SPV system with effective solution searching space. From the result of execution time, GA model shows rather competitive as compared to the Lagrange technique.

\section{Conflict of Interests}

The authors declare that there is no conflict of interests regarding the publication of this paper.

\section{Acknowledgments}

The authors would like thank the National Science Council of the Republic of China, Taiwan, for financially supporting this research under Contract no. NSC102-2221-E-218-019.

\section{References}

[1] G. C. Onwubolu and B. V. Babu, New Optimization Techniques in Engineering, Studies in Fuzziness and Soft Computing, Springer, New York, NY, USA, 2004.

[2] A. A. Bazmi and G. Zahedi, "Sustainable energy systems: role of optimization modeling techniques in power generation and supply-a review," Renewable \& Sustainable Energy Reviews, vol. 15 , no. 8, pp. 3480-3500, 2011.

[3] D. E. Goldberg, Genetic Algorithms in Search, Optimization, and Machine Learning, Addison-Wesley, 1989.

[4] G. Poonam, "A comparison between memetic algorithm and genetic algorithm for the cryptanalysis of simplified data encryption standard algorithm," International Journal of Network Security \& Its Application, vol. 1, no. 1, pp. 34-42, 2009.

[5] R. Baños, F. Manzano-Agugliaro, F. G. Montoya, C. Gil, A. Alcayde, and J. Gómez, "Optimization methods applied to renewable and sustainable energy: a review," Renewable and Sustainable Energy Reviews, vol. 15, no. 4, pp. 1753-1766, 2011.

[6] M. M. M. El-Arini, A. M. Othman, and A. Fathy, "A new optimization approach for maximizing the photovoltaic panel power based on genetic algorithm and Lagrange multiplier algorithm," International Journal of Photoenergy, vol. 2013, Article ID 481468, 12 pages, 2013.
[7] M. S. Ismail, M. Moghavvemi, and T. M. I. Mahlia, "Characterization of PV panel and global optimization of its model parameters using genetic algorithm," Energy Conversion and Management, vol. 73, pp. 10-25, 2013.

[8] A. Mellit, S. A. Kalogirou, L. Hontoria, and S. Shaari, "Artificial intelligence techniques for sizing photovoltaic systems: a review," Renewable and Sustainable Energy Reviews, vol. 13, no. 2, pp. 406-419, 2009.

[9] A. Arabali, M. Ghofrani, M. Etezadi-Amoli, M. S. Fadali, and Y. Baghzouz, "Genetic-algorithm-based optimization approach for energy management," IEEE Transactions on Power Delivery, vol. 28, no. 1, pp. 162-170, 2013.

[10] J. L. Bernal-Agustín and R. Dufo-López, "Simulation and optimization of stand-alone hybrid renewable energy systems," Renewable and Sustainable Energy Reviews, vol. 13, no. 8, pp. 2111-2118, 2009.

[11] A. Benatiallah, R. Mostefaoui, M. Boubekri, and N. Boubekri, "A simulation model for sizing PV installations," Desalination, vol. 209, no. 1-3, pp. 97-101, 2007.

[12] O. M. Toledo, D. O. Filho, A. S. A. C. Diniz, J. H. Martins, and M. H. M. Vale, "Methodology for evaluation of grid-tie connection of distributed energy resources-case study with photovoltaic and energy storage," IEEE Transactions on Power Systems, vol. 28, no. 2, pp. 1132-1139, 2013.

[13] R. Bakhshi, J. Sadeh, and H. R. Mosaddegh, "Optimal economic designing of grid-connected photovoltaic systems with multiple inverters using linear and nonlinear module models $\mathrm{b}$ ased on Genetic Algorithm," Renewable Energy, vol. 72, pp. 386-394, 2014.

[14] G. A. F. Seber, Linear Regression Analysis, John Wiley \& Sons, 1977.

[15] T. Seki, N. Yamashita, and K. Kawamoto, "New local search methods for improving the lagrangian-relaxation-based unit commitment solution," IEEE Transactions on Power Systems, vol. 25, no. 1, pp. 272-283, 2010. 


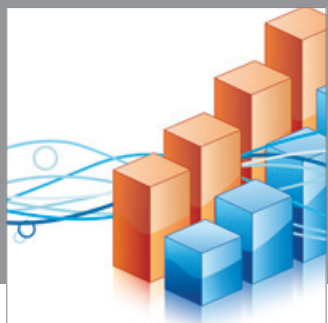

Advances in

Operations Research

mansans

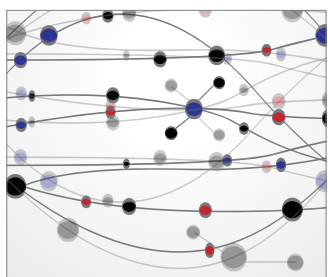

The Scientific World Journal
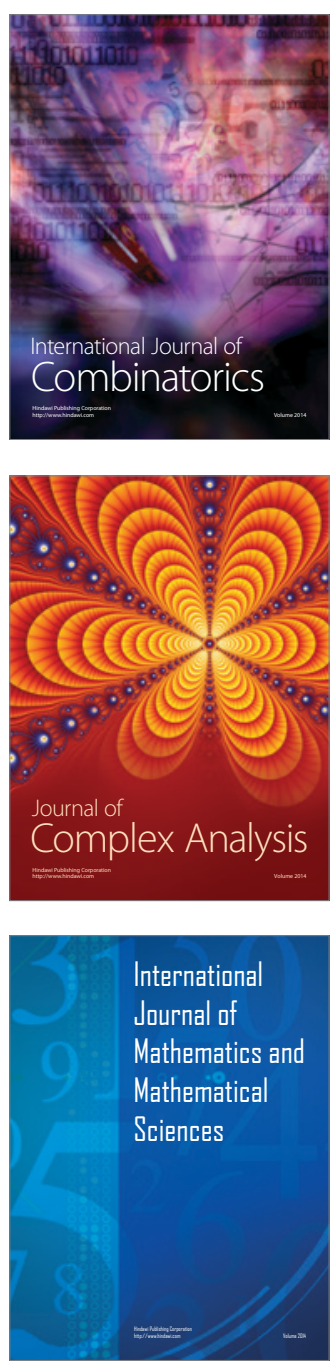
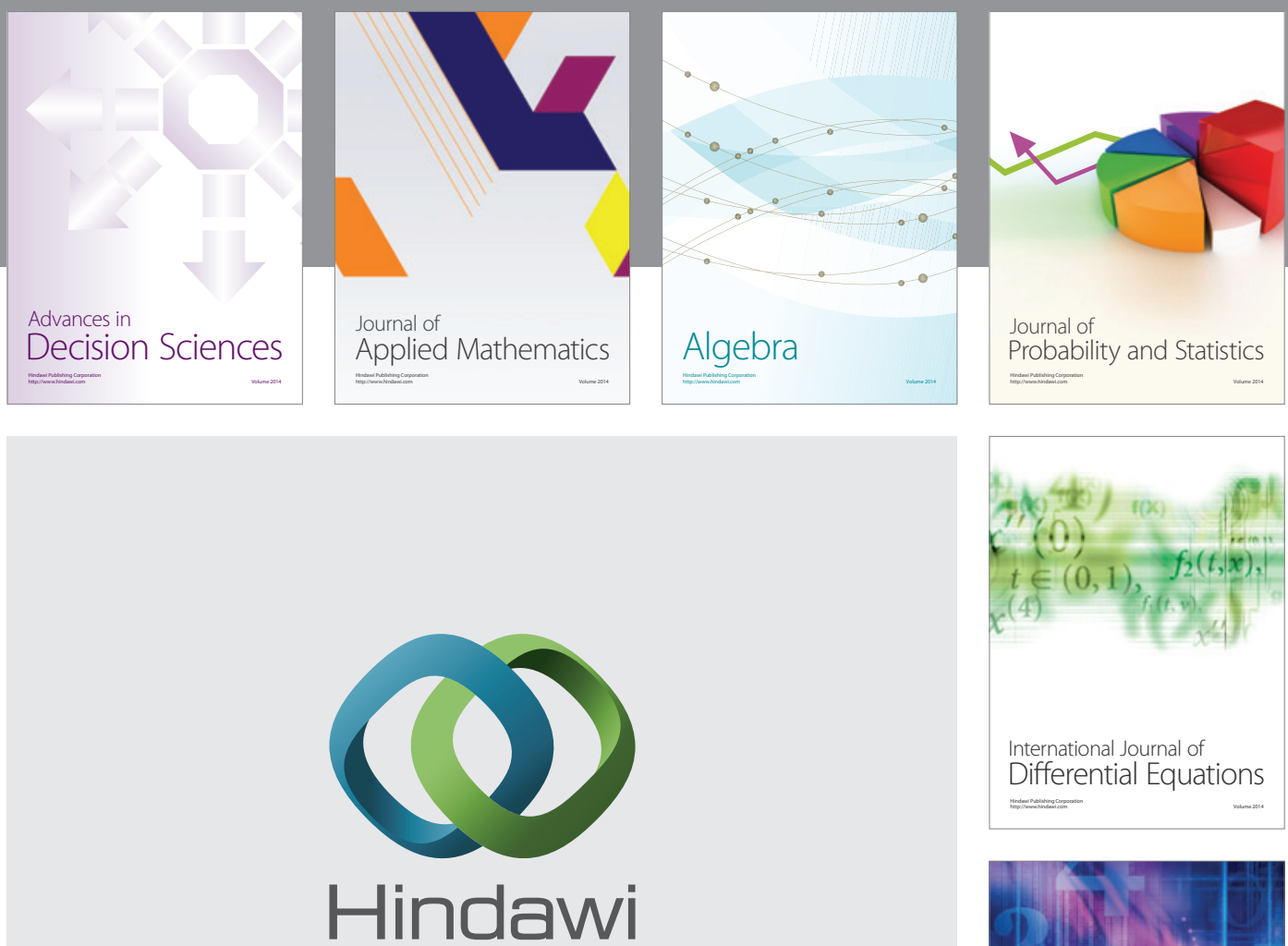

Submit your manuscripts at http://www.hindawi.com
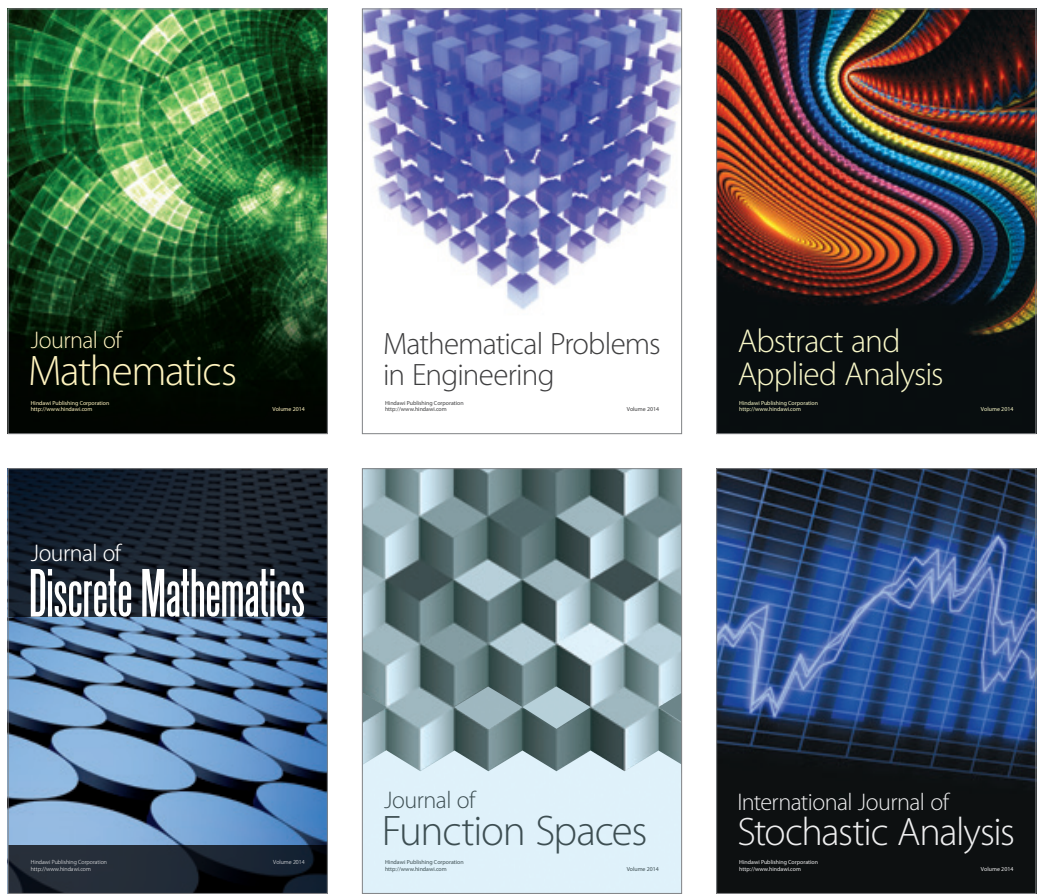

Journal of

Function Spaces

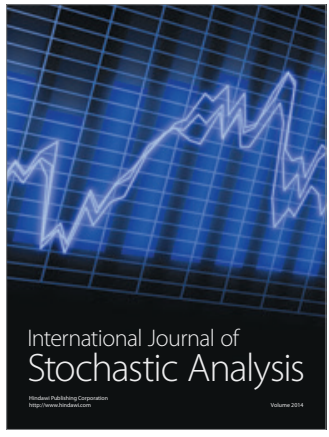


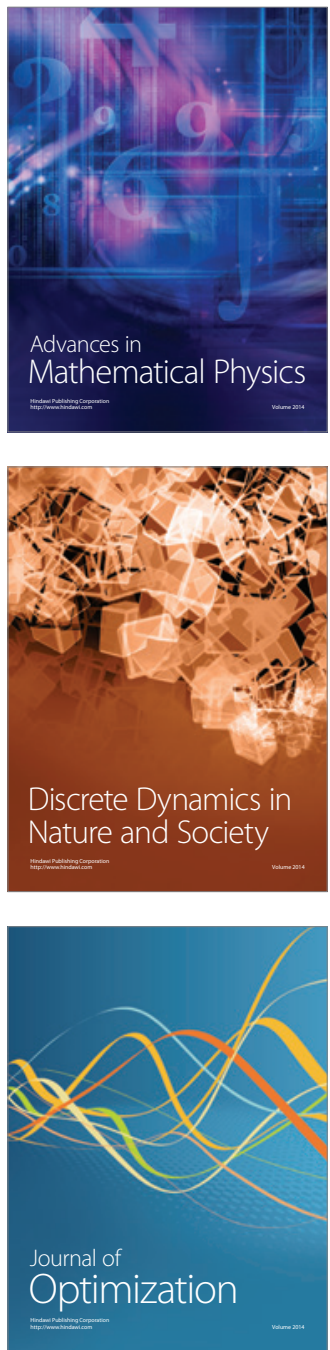